\title{
Stage IB Gastric Cancer AJCC v7
}

National Cancer Institute

\section{Source}

National Cancer Institute. Stage IB Gastric Cancer AJCC V7. NCI Thesaurus. Code C5469.

Stage IB includes: (T2, N0, M0); (T1, N1, M0). T1: Tumor invades lamina propria,

muscularis mucosae, or submucosa. T2: Tumor invades the muscularis propria. N0: No

regional lymph node metastasis. N1: Metastasis in 1-2 regional lymph nodes. M0: No

distant metastasis. (AJCC 7th ed.) 